\title{
Mean and turbulent mass flux measurements in an idealised street network
}

\author{
Matteo Carpentieri ${ }^{\mathrm{a}, *}$, Alan G. Robins ${ }^{\mathrm{a}}$, Paul Hayden ${ }^{\mathrm{a}}$, Edoardo Santi ${ }^{\mathrm{a}, \mathrm{b}}$ \\ ${ }^{a}$ EnFlo, Department of Mechanical Engineering Sciences, University of Surrey, Guildford, \\ GU2 7XH, UK \\ ${ }^{b}$ Dipartimento di Ingegneria Industriale, Università degli Studi di Firenze, Via di S.Marta \\ 3, 50139 Firenze, Italy
}

\begin{abstract}
Pollutant mass fluxes are rarely measured in the laboratory, especially their turbulent component. They play a major role in the dispersion of gases in urban areas and modern mathematical models often attempt some sort of parametrisation. An experimental technique to measure mean and turbulent fluxes in an idealised urban array was developed and applied to improve our understanding of how the fluxes are distributed in a dense street canyon network. As expected, horizontal advective scalar fluxes were found to be dominant compared with the turbulent components. This is an important result because it reduces the complexity in developing parametrisations for street network models. On the other hand, vertical mean and turbulent fluxes appear to be approximately of the same order of magnitude. Building height variability does not appear to affect the exchange process significantly, while the presence of isolated taller buildings upwind of the area of interest does. One of the most interesting results, again, is the fact that even very simple and regular geometries lead to complex advective patterns at intersections: parametrisations derived from measurements in simpler geometries are unlikely to capture the full complexity of a real urban area.
\end{abstract}

Keywords: Wind tunnel, Neighbourhood scale, Urban areas, Pollutant exchanges, Turbulent fluxes

\section{Capsule}

Wind tunnel measurements of mean and turbulent pollutant fluxes in street networks highlight the importance of geometrical details

\footnotetext{
* Corresponding author

Email address: m.carpentieri@surrey.ac.uk (Matteo Carpentieri)
} 


\section{Introduction}

Modelling air pollution in urban areas has become more and more important in recent years when potential health problems might arise from traffic emissions as well as accidental or deliberate releases of hazardous gases. Mathematical models for real urban areas should take into account a wide range of spatial and temporal scales. Computational fluid dynamics (CFD) models can adequately simulate small and medium sized urban regions (Tominaga \& Stathopoulos, 2013), but they have too time-intensive to be used for air quality management or rapid response in case of an emergency. For this purpose, fast approximate models are better suited to provide a rapid answer and they must be able to both parametrise the physical phenomena in a complex geometry and giving accurate and reliable results.

One of the many complications that exist in real urban areas is represented by street canyons that are often pollutant hot-spots. Contrary to the classical definition (infinitely long street with buildings on both sides), in actual cities their length is limited and they are not isolated, being connected to other streets at intersections. Dispersion in street canvon intersections is still rarely studied (see, e.g.. Hoydysh \& Dabberdt, 1994; Scaperdas, 2000; Carpentieri et al., 2009; Carpentieri \& Robins, 2010) compared to isolated canyons. Pollutant can be exchanged between several streets and with the flow above the canopy at urban intersections and a better knowledge of these transport processes is very important for developing reliable mathematical parametrisations. Real intersections, however, are often characterised by highly three-dimensional flows and are not easy to study in a systematic manner.

To make matters more complicated, both advective and turbulent mass exchanges play a significant role in transferring gases in and out of the street canyons (Caton et al., 2003; Salizzoni et al., 2011). Despite this, very few experimental studies of urban-like models (Carpentieri et al., 2012) focussed on turbulent fluxes, mainly because the simultaneous measurement of velocity and concentration in laboratory is very hard to achieve.

Fackrell \& Robins (1982), Zhu et al. (1988) and Lemoine et al. (1997) applied conventional measurement techniques, such as the combined use of hot wire anemometry (HWA) and tracer concentration fluctuation measurements, to quantify turbulent mass fluxes in wind tunnel experiments. The first use of conventional techniques to measure turbulent mass fluxes in an urban model. and in particular within street canyons and intersections, was that of Carpentieri et al. (2012), who combined laser Doppler anemometry (LDA) and fast flame ionisation detector (FFID) measurements to derive pollutant exchanges in a complex London neighbourhood.

Other authors derived alternative measurement techniques to be used in urban models. Dezső-Weidinger et al. (2003) used particle tracking velocimetry (PTV) to assess whether turbulent mass fluxes are proportinal to concentration gradients in a regular-shaped two-dimensional street canyon. They found that this common assumption does not hold in the canopy region. Integral mass flux calculations were not attempted in their study. A similar study in a water flume 
was carried out by Caton et al. (2003) with the aim of validating their pollutant mass exchange parametrisation, but they neglected turbulent fluxes.

Barlow \& Belcher (2002) used naphthalene sublimation to estimate spatially averaged total mass fluxes (see also Barlow et al., 2004; Pascheke et al., 2008), but this technique cannot separate the advective from the turbulent compoments. Similarly, Narita (2007) used water evaporation instead of naphthalene. Another similar approach would involve the use of heat as an analog for mass and measuring heat fluxes.

Urban dispersion models can range from simple street canyon approaches (e.g. ADMS-Urban. McHugh et al., 1997), to street network models (e.g. SIRANE, Soulhac et al., 2011), to full building-resolved CFD models. They all rely on some sort of parametrisation of the pollutant mass transfer within and above the street canopy, and these are particularly important for street network models (Ben Salem et al., 2015; Belcher et al., 2015). There is a need, then, to produce experimental data sets that can be used to validate these parametrisations (Gamel et al., 2015).

This and the results from previous wind tunnel experiments (Carpentieri et al., 2009; Carpentieri \& Robins, 2010) motivated the development a robust technique to measure both advective and turbulent mass fluxes and be able to assess their individual contributions. This technique was developed and preliminarily tested in the DAPPLE model by Carpentieri et al. (2012). The application of simultaneous LDA and FFID measurements proved extremely useful in assessing pollutant fluxes around street intersections, but the geometrical complexity of the model posed many obstacles, first of all the fact that, because of the difficulties in identifying a "roof level" when all buildings heights were different, a vertical pollutant exchanges between the urban canopy and flow above could not be directly measured but only indirectly estimated. A similar technique, with a coupled HWA-FFID method, was subsequently applied to the flow around a 2D obstacle in the detailed study by Gamel (2015) and Gamel et al. (2015), but no measurements were made in more complex settings such as street canyons or intersections.

The present study uses the technique developed by Carpentieri et al. (2012), but the wind tunnel experiments are applied to more regular models of idealised urban street networks. The same technique was applied to more complex urban arrays by Nosek et al. (2016) and Nosek et al. (2017). Their results, while interesting, are not directly comparable with the present study due to the large differences in the studied geometry, especially as far as the roofs are concerned.

In the present study, the measurements are taken around a central intersection where all the buildings have the same height, thus allowing a direct measurement of vertical exchanges. The study is part of the HRModUrb project (Carpentieri, 2013) and follows up flow measurements and numerical simulations carried out to assess the influence of urban morphology (in particular building height variability) on flow and dispersion in urban street networks (Carpentieri \& Robins, 2015).

The main objectives of this work are: (i) to study the feasibility of using this technique to measure pollutant fluxes in complex urban models; (ii) to 
understand the processes driving vertical and horizontal pollutant exchanges in building arrays; and (iii) to derive integral mass exchange balances.

\section{Experimental setup}

The two idealised urban models used in this study were described in detail by Carpentieri \& Robins (2015). They were developed using morphological parametres from the central region of the DAPPLE site in London, in particular as far as building area density $\left(\lambda_{p}=0.54\right)$ and mean building heigth $\left(H_{b}=102\right.$ $\mathrm{mm}$ ) are concerned. "SimpleC" is the first model, with all buildings of the same height. "SimpleV", the second model, has the same building density and mean height, but it includes blocks with different heights. The sandard deviation of the building heights is the same as in the DAPPLE site $\left(\sigma_{H}=32 \mathrm{~mm}\right)$ as well as the frontal area densities for the two wind directions $\left(\lambda_{f}=0.25\right.$ along the $x$ axis, $\lambda_{f}=0.16$ along the $y$ axis). Further details can be found in Carpentieri \& Robins (2015) and supplemental material.

The EnFlo boundary layer wind tunnel at the University of Surrey, where the experiments were carried out, is an open circuit wind tunnel (test section: $20 \mathrm{~m} \times 3.5 \mathrm{~m} \times 1.5 \mathrm{~m}$ ). The reference flow velocity is measured by an ultrasonic anemometer outside the generated boundary layer. Tracer concentrations were measured by a fast response Flame Ionisation Detector (FFID), while velocities were investigated by means of a two-component Laser Doppler Anemometer (LDA). This setup allows the simultaneus measurements of concentration and velocity, as described by Carpentieri et al. (2012), thus enabling the estimation of both the mean and turbulent scalar fluxes. The averaging time was around $70 \mathrm{~s}$ in most measurements, so that typical values for the standard errors were around $2 \%$ for the mean velocity, the vertical velocity variance and both the concentration mean and variance, and around $3 \%$ for the horizontal velocity variance.

For this series of experiments, two model orientations were tested: $90^{\circ}$ (wind aligned with the $y$ axis) and $45^{\circ}$. The experiments were performed using a reference wind speed $U_{\text {ref }}=2.0 \mathrm{~m} \mathrm{~s}^{-1}$. The approach flow obtained is fully described by Carpentieri \& Robins (2015). Roughness length and friction velocity were estimated from the logarithmic profile fit: $z_{0}=0.015 H_{b}$ and $u_{*}=0.06 U_{\text {ref }}$. The measurements were located around the central intersection covering an horizontal layer at roof level (figure 1a), and the inlet and outlet vertical sections of the 4 streets around the intersection (figure $1 \mathrm{~b}$ and 1c), covering the volume of interest with a reasonably high spatial resolution. The present study is mainly concerned with scalar fluxes, so for each measurement section (surface), the relevant velocity component has been measured: that includes the $W$ component in horizontal sections (i.e. vertical component through the roof level areas), the $U$ component in vertical sections perpendicular to $x$ (i.e. horizontal fluxes along X-street) and the $V$ component in vertical sections perpendicular to $y$ (i.e. horizontal fluxes along Y-street).

The source for the tracer measurements was located as shown in figure 2 so that at the intersection we could claim to be in the intermediate-field regime 

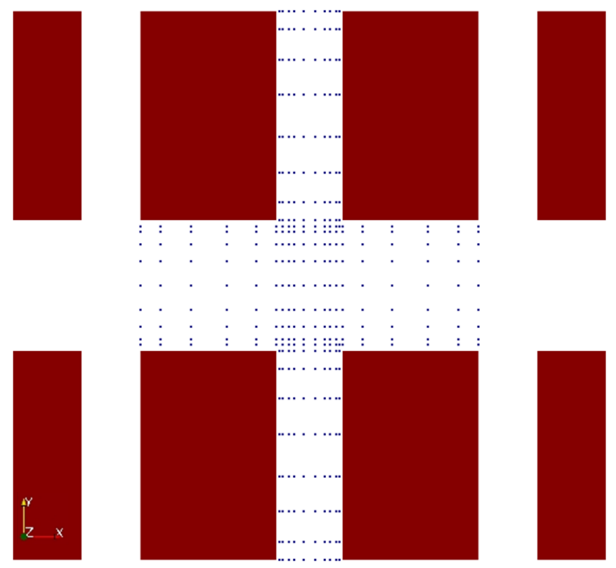

(a) Top view (XY plane)
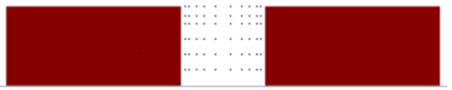

(b) Side view (XZ plane)

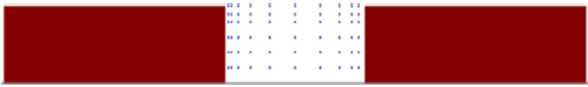

(c) Side view (YZ plane)

Figure 1: Flux measurement points for (a) vertical flux at roof level, (b) horizontal flux along $\mathrm{Y}$ street and (c) horizontal flux along $\mathrm{X}$ street

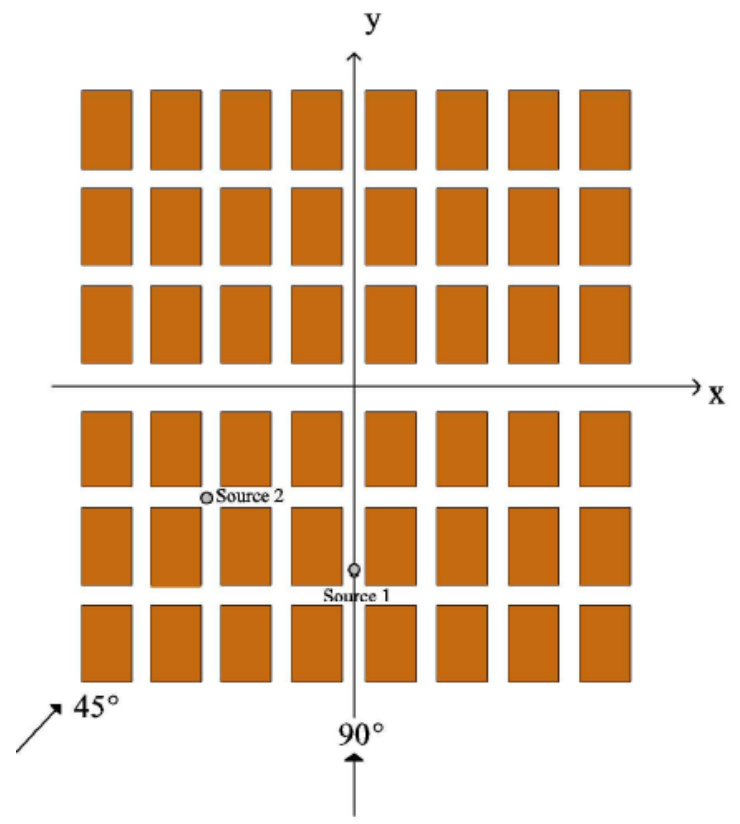

Figure 2: Location of the sources for the $90^{\circ}$ (Source 1) and $45^{\circ}$ (Source 2) cases 
(a few street canyons downstream in all cases). Measuring mass fluxes in the far-field is, in fact, not very useful, as concentrations would be uniform across the different regions. While the ultimate goal is to be able to measure mass fluxes and produce reliable exchange balances for the near-field regime, we have decided to test our methodology in an easier situation. In the intermediate-field dimensions of the dispersion plume are already of the same order of magnitude as the buildings, but the concentrations are not as uniform as in the far-field. Given the challenges we faced with this configuration (see sections 4 and 5), this proved to be a wise decision, as it is unlikely that the present technique could be used to provide reliable mass flux balances for the near-field without substantial improvements. The tracer gas was a mixture of a small amount of propane in air, with a flow rate $Q=4.0 \times 10^{-5} \mathrm{~m}^{3} \mathrm{~s}^{-1}$ and it was released from a diffuser with exit internal dimensions of $70 \mathrm{~mm}$ (width) by $8 \mathrm{~mm}$ (height) placed at ground level across the street. The target value for the flow rate is a compromise between having a reliable source release, detectable in the study area, and a low emission velocity, so as to have a passive release with no more than the maximum allowable (for health and safety reasons) ratio of propane in air $(1.8 \%)$. Porous material was used within the outlet section of the diffuser to ensure a passive and uniform release.

$U$ and $W$ velocity components were measured at roof level by using the LDA alone by Carpentieri \& Robins (2015). In this study, they were measured by installing both the LDA and FFID probes in the wind tunnel. A comparison of the two data sets was made in order to validate the velocity measurements with the latter set-up. The relative differences for $U$ component velocity appear to be quite small. The average difference was found to be around $6 \%$, with $86 \%$ of the measurements differing by less than $10 \%$. The percentage differences are much higher for the $W$ component, but this was partly expected given the very low absolute values of velocities involved. Good comparability was also found for turbulence data, with velocity variances differing $13 \%$ ( $u$ component) and $8 \%(w)$ on average.

\section{Scalar fluxes}

\subsection{Exchanges at roof level}

Measurements of mean and turbulent exchanges are reported in terms of local non-dimensional fluxes, that is:

$$
\Phi_{i}^{*}=\frac{\overline{U_{i}} \overline{C^{*}}}{U_{\text {ref }}}+\frac{\overline{u_{i} c^{*}}}{U_{\text {ref }}}
$$

where $C^{*}$ and $c *$ are the non-dimensional concentration and concentration fluctuation, calculated as:

$$
C^{*}=\frac{C U_{r e f} H_{b}^{2}}{Q} ; \quad c^{*}=\frac{c U_{r e f} H_{b}^{2}}{Q}
$$


with $Q=$ source flow rate and the subscript $i$ indicates different velocity components. Two components of the flux can be distinguished in equation 1: the advective component (left-hand side addend) and the turbulent component (righthand side addend).

Figures 3 - 4 show maps of total, mean and turbulent vertical fluxes through the roof level cross-sections. In all cases, turbulent and mean vertical flux values at roof level were found to be approximately of the same order of magnitude, even though differences can be seen in different experimental conditions. In general, advective fluxes seem to have larger variations (either positive or negative) compared with the turbulent ones.

When the wind is blowing parallel to the $\mathrm{Y}$ street $\left(90^{\circ}\right.$ cases, figure 3) the advective flux is generally very close to 0 , as expected, with large regions of positive and negative fluxes around the main intersection. A slight asymmetry can be observed in the SimpleC case. This is probably due to slight misalignments in the building blocks that are unavoidable in a complex experimental setting such as the present one (see also Castro et al., 2017, for a detailed discussion on this point). As confirmed by the numerical data, the turbulent flux is mainly from the canopy upwards, resulting in an overall slight positive total flux in the $\mathrm{Y}$ street, while in the lateral (X) streets the total flux is close to zero or slightly negative due to advection.

The SimpleV case (model with variable building heights) is interesting if compared to the SimpleC one. While the general patterns observed for the constant height array still hold (advective fluxes very small and slighlty positive turbulent flux of the same order of magnitude as the mean flux), larger positive advective fluxes can be observed in the upwind part of $\mathrm{Y}$ street. This is probably due to the mean flow driven by the taller buildings just upwind of the intersection. The turbulent fluxes are slightly smaller than in the SimpleC case, probably due to the slower flow (Carpentieri \& Robins, 2015) caused by the already mentioned upwind tall buildings. The asymmetric pattern highlighted in the SimpleC case is less evident here. A possible explanation is that the SimpleV model is more affected by the flow above the canopy, and that makes it less sensitive to small misalignments in the building blocks. As mentioned, a more detailed discussion on the various sources of error is presented in section 5 .

$45^{\circ}$ cases (figure 1 ) present a more complex behaviour, with the general positive flux interrupted by regions of negative flux in the wake of the buildings (similar to what Castro et al., 2017, have found in their LES simulations). In these cases the small positive turbulent flux seems to be smaller compared to the advective component than in the other cases. Building height variability (SimpleV) does not seem to affect the flux throught these roof level sections. Any difference is within the expected experimental uncertainty. In fact, in the $45^{\circ}$ configuration, the two tall buildings are relatively out of the flow path through the central intersection, while the other buildings (mainly the "green" and "yellow" blocks, slightly less tall than the central intersection) do not seem to affect the vertical exchange much despite their different height. This supports the fact that appreciably taller buildings upwind of the observed area can 


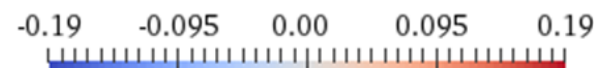

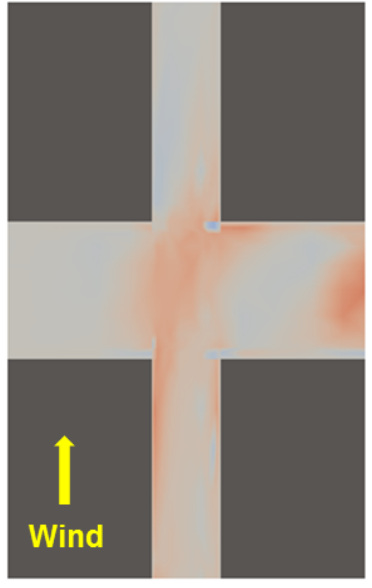

(a) SimpleC, total

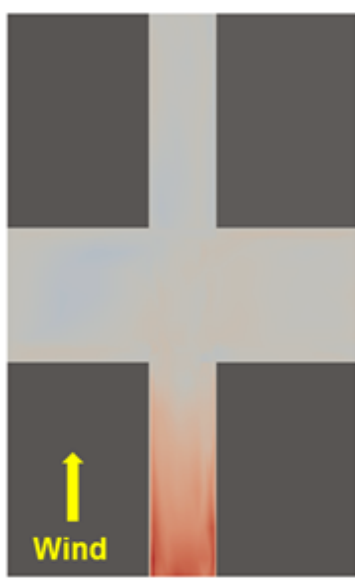

(d) SimpleV, total

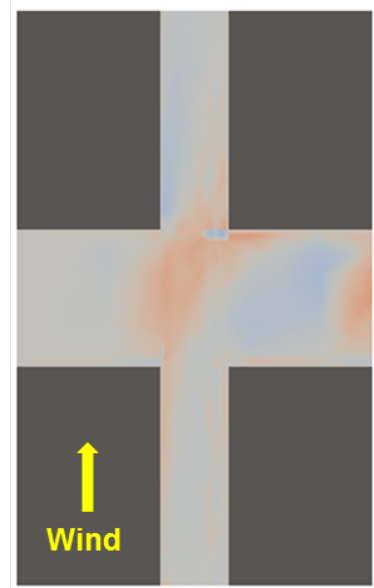

(b) SimpleC, mean

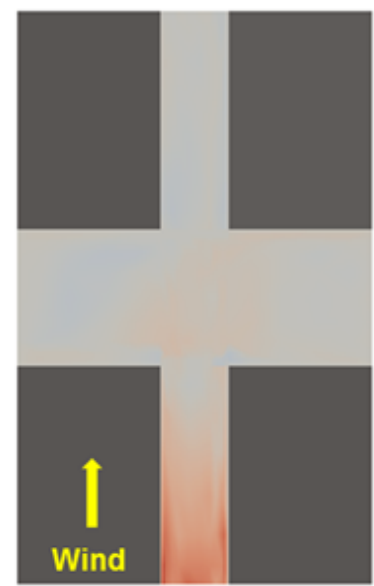

(e) SimpleV, mean

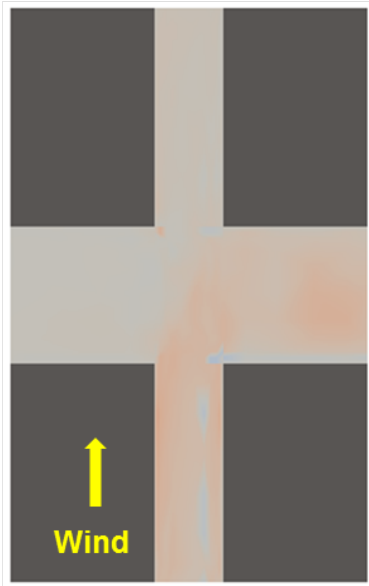

(c) SimpleC, turbulent

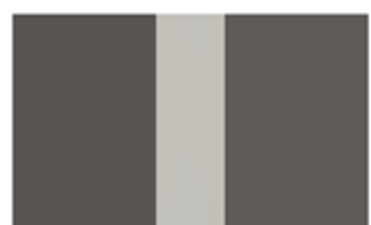

Wind

(f) SimpleV, turbulent

Figure 3: Non-dimensional vertical flux, $90^{\circ}$ case 


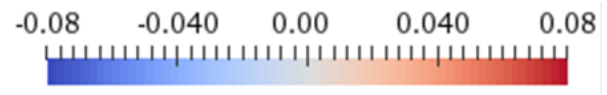

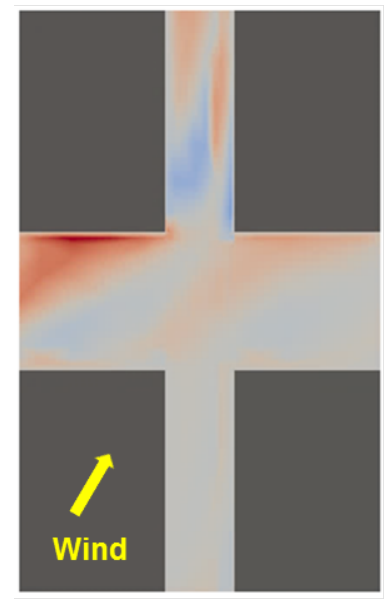

(a) SimpleC, total

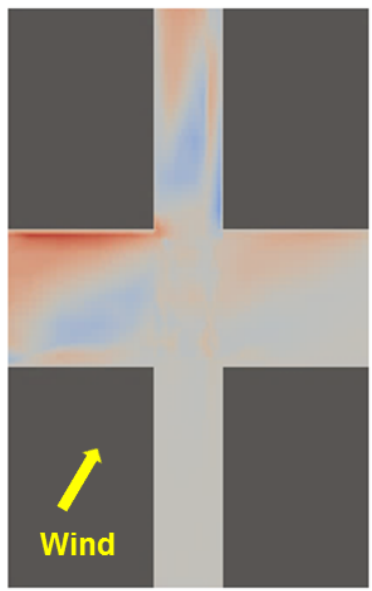

(d) SimpleV, total

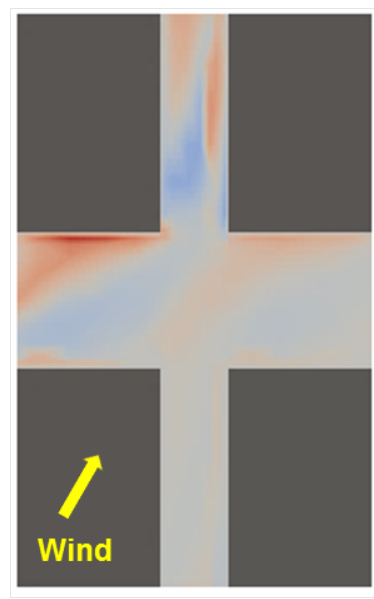

(b) SimpleC, mean

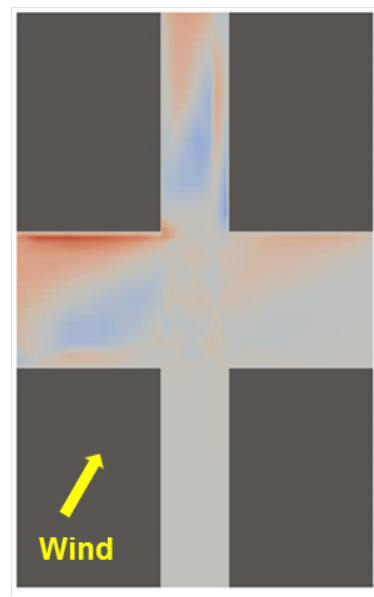

(e) SimpleV, mean

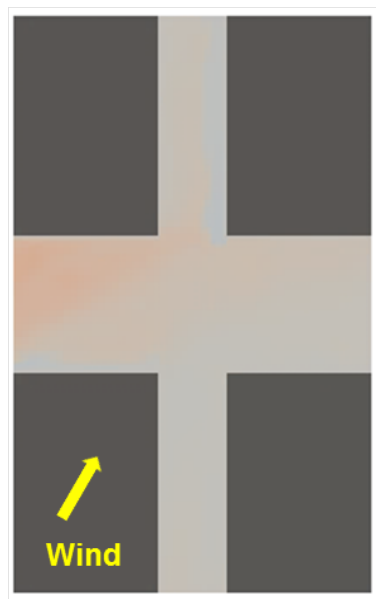

(c) SimpleC, turbulent

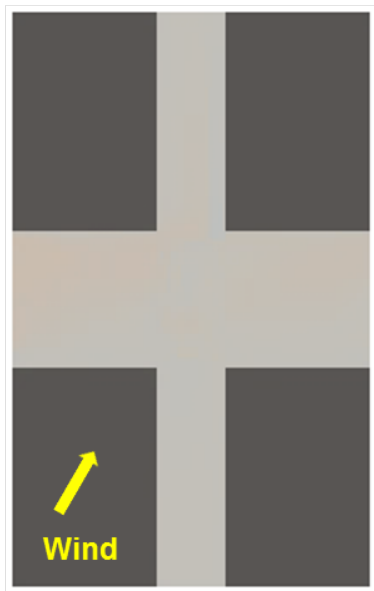

(f) SimpleV, turbulent

Figure 4: Non-dimensional vertical flux, $45^{\circ}$ case; the scale is different than figure 3 to highlight the flux distribution in this particular case 


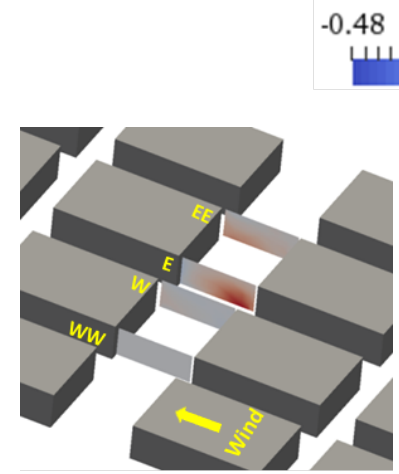

(a) SimpleC, total

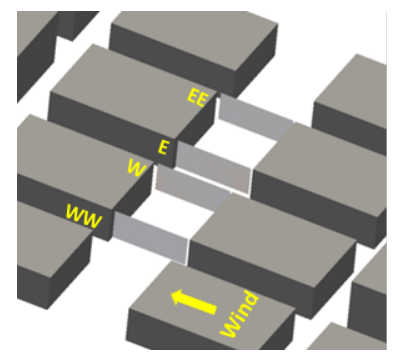

(d) SimpleV, total

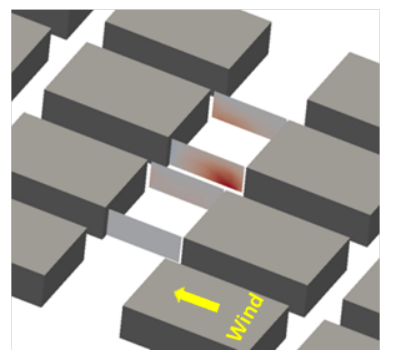

(b) SimpleC, mean

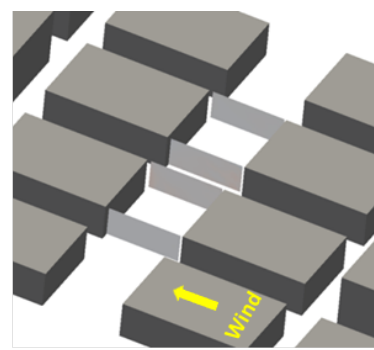

(e) SimpleV, mean

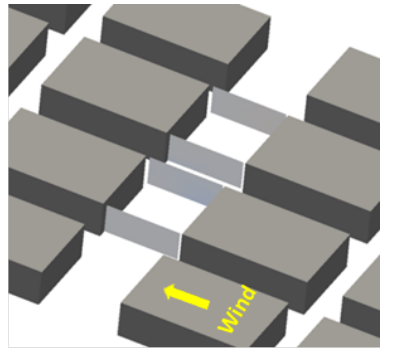

(c) SimpleC, turbulent

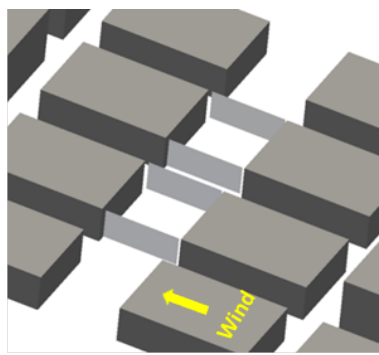

(f) SimpleV, turbulent

Figure 5: Non-dimensional horizontal flux in $\mathrm{X}$ street, $90^{\circ}$ case

have a disproportionate effect on the dispersion mechanisms compared to other grometric features of an urban area.

These results also highlight the horizontal variability of the vertical fluxes in the measurement region. Single vertical profiles of mass fluxes or a single vertical section along (or across) the street canyon - as it is often the case in previous studies in the literature - are unlikely to capture the full complexity of the intersection, even for relatively simple and regular street canyons, as in the present case. The exact location of the measurement may also affect the observations, given the large gradients measured in some cases: in both the $90^{\circ}$ and $45^{\circ}$ cases the total flux might be either positive or negative within a very short distance (less than $15 \%$ of the street width) from the centre of the intersection.

\subsection{Exchanges between streets and intersection}

Some selected vertical cross-sections of mean, turbulent and total horizontal fluxes are shown in figures $5-6$.

Horizontal fluxes along $\mathrm{Y}$ street in the $90^{\circ}$ case are obviously mostly due to advective terms, so they are not shown in the figures. Turbulent flux fields are at least 1 or 2 orders of magnitude smaller and generally directed downstream (positive $y$ direction). Exchanges with the lateral streets (X street) are 
$\begin{array}{lllll}-0.19 & -0.095 & 0.00 & 0.095 & 0.19\end{array}$

шسшس

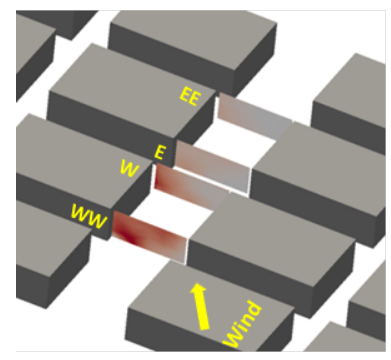

(a) SimpleC, total

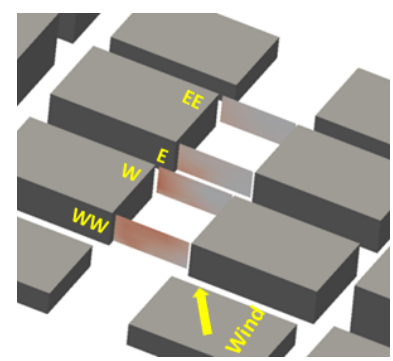

(d) SimpleV, total

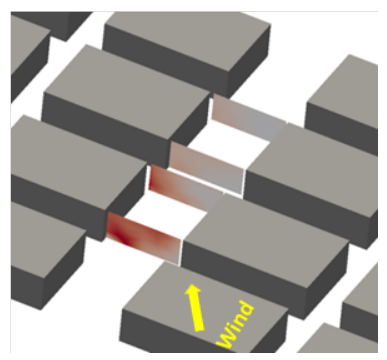

(b) SimpleC, mean

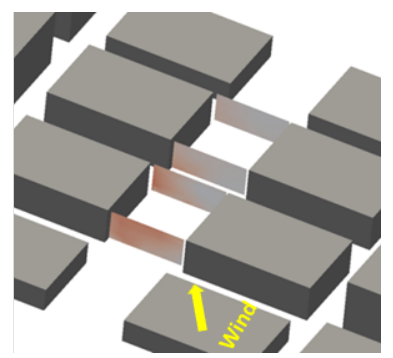

(e) SimpleV, mean

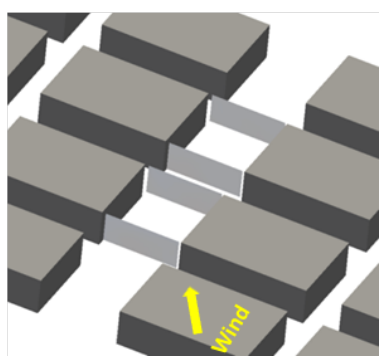

(c) SimpleC, turbulent

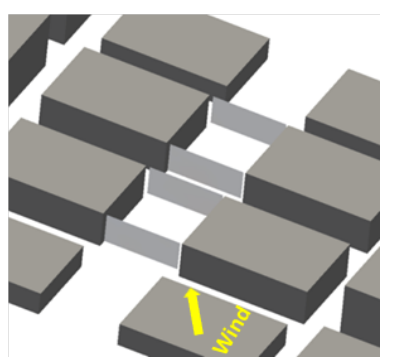

(f) SimpleV, turbulent

Figure 6: Non-dimensional horizontal flux in $\mathrm{X}$ street, $45^{\circ}$ case 
quite limited, and mostly due to advective terms (recirculations) in the main intersection (figure 5). The asymmetry highlighted in section 3.1 can be clearly seen in the figure (see also section 5). Apart from this aspect no other obvious differences can be observed between SimpleC and SimpleV.

For the $45^{\circ}$ case, most of the exchange process occurs due to advective terms. No differences in the general behaviour of the horizontal fluxes can be found between the SimpleC cases and the SimpleV cases.

These results confirm the fact that horizontal turbulent exchanges between streets can be generally neglected in a street network, except in very special cases. For example, when the wind is perfectly aligned with one street and perpendicular to the other the turbulent flux becomes as important as the mean flux. In that case, though, the total flux is so small that it can itself be neglected; also, one might point out that this case is not very realistic anyway, since a perfectly parallel wind in a perfectly orthogonal street network does not happen very often in reality. As soon as there is a slight deviation from the ideal situation the advective flux becomes predominant and the turbulent flux can be safely ignored, as the anomalous asymmetric observation in the $90^{\circ} \mathrm{SimpleC}$ case demonstrates. The present results are also in line with the findings by Carpentieri et al. (2012) in a more complex urban setting (where "ideal" was hardly a feature).

\section{Tracer flux balances}

The overall tracer flux, $\Phi_{S}$, normal to a reference plane $S$ can be calculated as:

$$
\Phi_{S}=\iint_{S} \Phi_{n}^{*} \mathrm{~d} s
$$

Every section used for the calculation, either vertical or horizontal (see section 2), was divided into cells defined within a uniform grid spaced $0.36 \mathrm{~mm}$ $\times 0.36 \mathrm{~mm}$. The flux through each cell was calculated by means of a spline interpolation on the measured values multiplied by the cell area. Fluxes at points on the ground or on the building walls were set to 0 . The overall fluxes (either advective, turbulent or total) through the considered section were then calculated by summing the fluxes through the corresponding cells. This methodology is more accurate than the simpler linear interpolation used by Carpentieri \& Robins (2010) and Carpentieri et al. (2012).

As explained in section 2, eight vertical sections (horizontal fluxes) and five horizontal sections (vertical fluxes) were selected for the measurements. This resulted in a total of five different calculation volumes, where a tracer flux balance could be attempted: $\mathrm{X}$ street $\mathrm{W}$ (west of the intersection, negative $x$ ), $\mathrm{X}$ street $\mathrm{E}$ (east of the intersection, positive $x$ ), Y street $\mathrm{S}$ (south of the intersection, negative $y$ ), $\mathrm{Y}$ street $\mathrm{N}$ (north of the intersection, positive $y$ ) and the central intersection itself. In the analysis, the vertical sections were labelled as follows: Xst WW (west end of X street W), Xst W (interface between X street $\mathrm{W}$ and the intersection), Xst $\mathrm{E}$ (interface between the intersection and $\mathrm{X}$ 
street E), Xst EE (east end of X street E), Yst SS (south end of Y street S), Yst $\mathrm{S}$ (interface between $\mathrm{Y}$ street $\mathrm{S}$ and the intersection), Yst $\mathrm{N}$ (interface between the intersection and $\mathrm{Y}$ street $\mathrm{N}$ ) and Yst NN (north end of $\mathrm{Y}$ street $\mathrm{N}$ ). The five horizontal sections were labelled according to the corresponding calculation volume: Top W (X street W), Top int (intersection), Top E (X street E), Top $\mathrm{S}$ (Y street $\mathrm{S}$ ) and Top N (Y street N).

The horizontal flux values of the ratios between turbulent and advective fluxes for the $90^{\circ}$ cases (wind parallel to the narrower Y street) are quite similar between the SimpleC and SimpleV models (please see supplemental material for a complete table). Turbulent along-wind components ('Yst' sections) are generally one to two orders of magnitude less than the mean fluxes and generally directed downwind. These fluxes tend to be smaller in the SimpleV case (section 3.2) as the air flow is slower. As far as the fluxes perpendicular to the wind ( $y$ component, 'Xst' sections) are concerned, the situation is more complex. Turbulent fluxes in this case are generally of the same order of magnitude as the advective fluxes (Xst WW, Xst W in Simple C and Xst EE in SimpleV), or one order of magnitude smaller (Xst E, Xst W SimpleV and Xst EE SimpleC). It must be noted, however, that in this case the quantities involved are actually very small, with almost negligible measured velocities, and a small uncertainty in the flux values can lead to very large errors in the integral flux calculation. Since the total flux is small, though, this does not strongly affect the overall balance calculation.

Vertical turbulent fluxes are of the same order of magnitude as the advective fluxes. The ratio between the two components is generally smaller in the Sim$\mathrm{pleV}$ case, which is probably due to an increase in advective fluxes from the greater complexity of the three-dimensional flow field (figure 3), as evidenced also in previous studies (Carpentieri et al., 2009).

In the $45^{\circ}$ cases, turbulent fluxes play a less significant role in the transfer process compared to advective fluxes, as is largely to be expected for the horizontal fluxes (see also Carpentieri et al., 2012). However, vertical turbulent fluxes seem to be smaller as well, compared to the $90^{\circ}$ cases (figure 4 ), but they are still a significant part of the total exchange process.

The overall tracer balances for the whole calculation volume (including the four canyons and the intersection) is shown graphically in figures 7 -8. For each case, the balance is reported in terms of total incoming flux $(=100 \%)$.

One point to be highlighted is the relatively high value of imbalances between incoming and outgoing fluxes: $11.9 \%$ of the flux is unaccounted for in the SimpleC $90^{\circ}$ case, $23.9 \%$ and $25.2 \%$ respectively for SimpleC and SimpleV in the $45^{\circ}$ case, and especially $35.8 \%$ in the SimpleV $90^{\circ}$ case. Looking at each single control volume (i.e. the four canyons and the central intersection), things look much better, with imbalance values lower than $5 \%$. The only values larger than $15 \%$ are those for the Xst $\mathrm{W}$ volume for the $45^{\circ}$ cases, respectively $16.1 \%$ (SimpleC) and $16.3 \%$ (SimpleV), and of course the SimpleV at $90^{\circ}$ case where the only anomalous value is a $21.4 \%$ imbalance for the Yst $\mathrm{S}$ volume. A more detailed discussion of this point can be found in section 5. Given these uncertainties in the results (see also the discussions in Carpentieri \& Robins, 2010; 

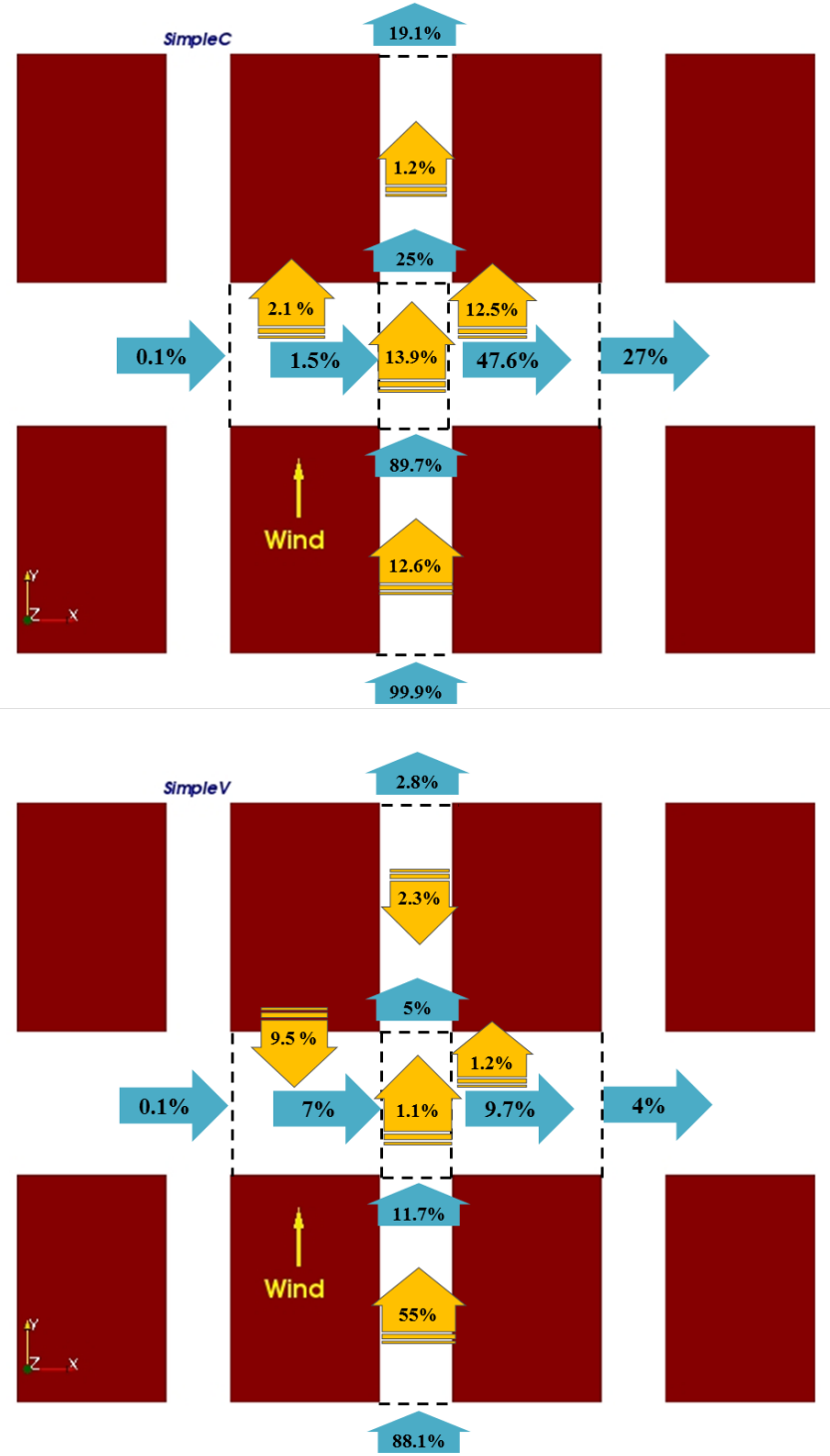

Figure 7: Overall tracer flux balance; $90^{\circ}$ wind direction, models SimpleC (top) and SimpleV (bottom); orange $=$ vertical exchange, blue $=$ horizontal 


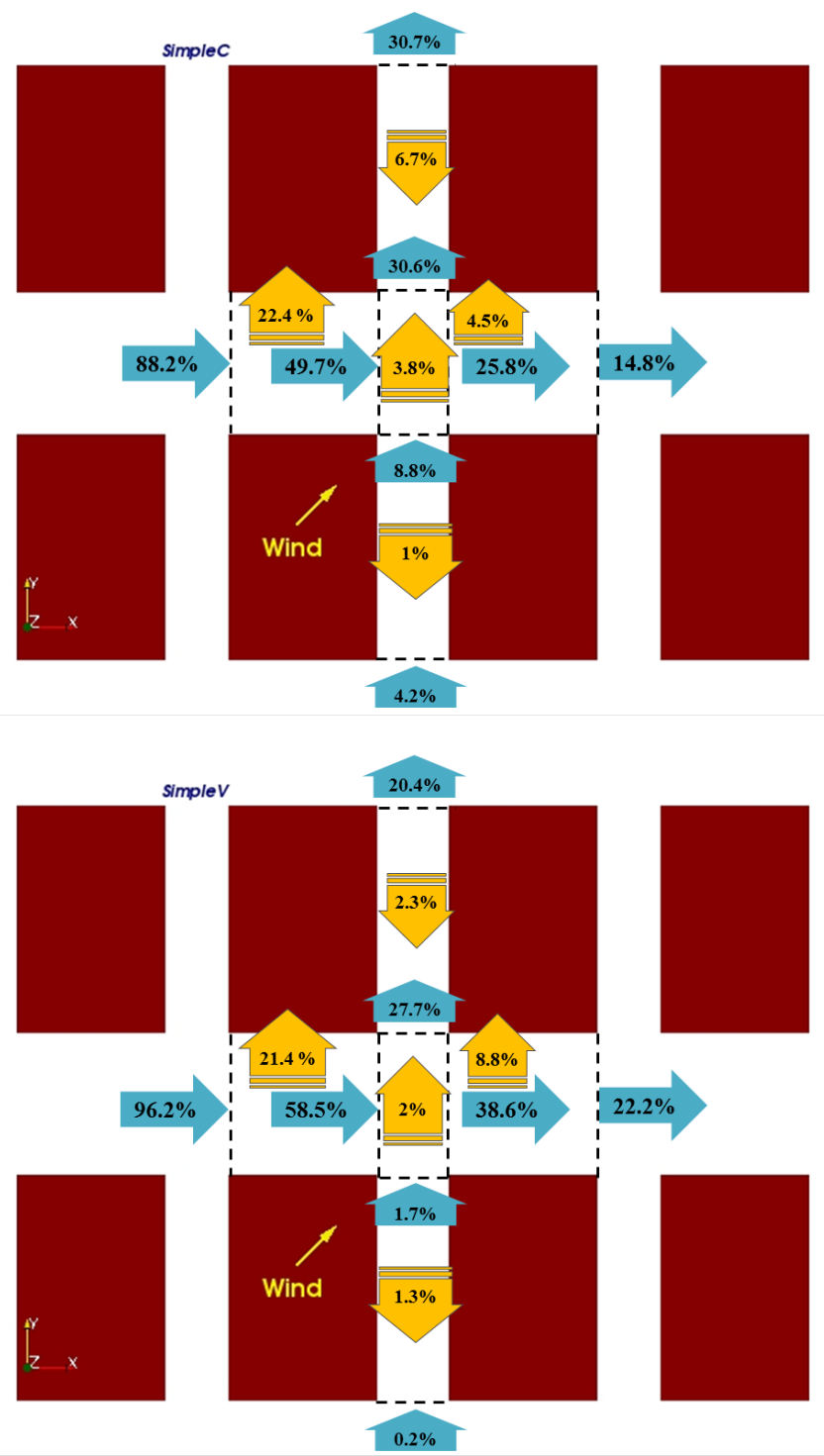

Figure 8: Overall tracer flux balance; $45^{\circ}$ wind direction, models SimpleC (top) and SimpleV (bottom; organge $=$ vertical exchange, blue $=$ horizontal) 
Carpentieri et al., 2012), the percentage values reported in the figures should be used mainly comparatively rather than as absolute values. This results in something more than a qualitative analysis, but certainly less than a completely quantitative one.

In the SimpleC $90^{\circ}$ case the flux is of course mostly coming from the south along the narrow central street. Within the control volumes this incoming flux is roughly equally split between one part that is continuing along the street canyons (with a very large portion of it spreading towards the right canyon because of the asymmetry noted in the previous sections) and one part detrained into the flow above roof level. In the SimpleV case the general trend is similar, but the proportion of flux detrained upwards, especially in the upwind canyon (Yst S), is much larger compared to the SimpleC case. This is probably due to the more complex 3D mean flow patterns highlighted both in section 3.1 and in Carpentieri \& Robins (2015). This is the first measured evidence of the influence of height variability on the pollutant exchange mechanism in urban canopies or, more precisely, the influence of taller buildings located upwind.

The situation is slightly different for the $45^{\circ}$ cases. In both the SimpleC and SimpleV models the majority of the flux is directed horizontally within the street canyons while a smaller, but still significant, fraction is detrained upwards. Differences between SimpleC and SimpleV are minimal in this case where no taller building is present along the wind bath, another illustration that the exchange process is driven more by the presence of upwind tall buildings than by height variability.

\section{Discussion and uncertainties}

\subsection{Flux measurement technique}

Results form this study and earlier measurements on the DAPPLE model (Carpentieri et al., 2012) have proven the reliability and usefulness of the described methodology for measuring turbulent mass fluxes. Observed values do not suffer from experimental uncertainty levels much greater than other measurement techniques (i.e., LDA alone or tracer concentration measurements alone) in terms of repeatability. Point measurements of turbulent and advective mass fluxes can be extremely useful for model validation purposes and to assess the relative importance of turbulent versus advective exchange processes (see, for example, Belcher et al., 2015).

The technique used in this study to measure concentrations and velocities simultaneously relies on coupling LDA and FFID. While the LDA probe is non-intrusive (the measurement volume is located at a distance of tens of $\mathrm{mm}$, depending on the lens), the FFID probe includes a small metal tube $(0.56 \mathrm{~mm}$ external diameter) that samples the air by pumping a small amount of air into the measuring device. In order not to disturb the velocity measurements, the inlet of the FFID probe was placed at a distance of $2 \mathrm{~mm}$ from the centre of the LDA measurement volume. Preliminary tests showed that the accuracy of the LDA measurements was only slightly affected by this arrangement. A more 
detailed analysis carried out by Gamel (2015), using the same measurement technique, confirms our findings.

On the other hand, the LDA measurement system needs particles to be injected into the flow and this might affect concentration measurements. The EnFlo LDA system employs micron sized sugar particles to seed the flow and their effect on the measured concentrations was found to be completely negligible. Gamel (2015) in his experiments found the FFID measurements slightly more affected by the particle seeding, but in that case the author was using vegetable oil, which can be more easily detected by the FFID giving a false concentration reading.

Since the sampled air volume needs some time to travel from the FFID probe inlet to the measuring device, LDA readings and concentration values must be properly synchronised. The sampling frequency of the FFID was greater than that of the LDA; this and the fact that LDA readings are irregular (they depend on detecting seeding particles passing through the measurement volume) meant that the LDA signal was the leading device for resampling the FFID signal. However, the resampling had to take into account the delay due to the travel time to the FFID measuring device. Choosing the correct value for this delay time is not trivial. Fluid dynamic considerations and maximisation of the correlation between velocity and concentration readings led us to choose a delay value of $25 \mathrm{~ms}$. This is roughly in line with the findings by Gamel (2015), who found a delay value in the range of 12 to $24 \mathrm{~ms}$, with an optimum for their system at $16 \mathrm{~ms}$.

\subsection{Spatial resolution and positional accuracy}

Despite the reliability of the measurement technique, integration of point data to calculate mass balances remains a challenge in the current context. Results reported here, as well as those by Carpentieri et al. (2012), can only be used qualitatively and it is clear that the quantitative values should be interpreted with care. Mass in and out was never balanced in the calculations, with large discrepancies in some cases. This problem was already highlighted by Carpentieri et al. (2012), but in the current study we reduced the potential causes. In fact, the studied geometry is much simpler than in the DAPPLE study and, more importantly, vertical fluxes through the top of the canyons and intersection have been measured. The remaining issues, then, as far as mass flux integration is concerned, is spatial positioning and resolution.

Getting a perfect positioning in the wind tunnel can be challenging. Given the large dimensions of the wind tunnel, expecting its floor to be perfectly even is unrealistic and, when measuring at a precise critical height, as in the present case, even a small deviation of, say, half a millimetre from the prescribed height can lead to large variations in critical locations. The positioning problem when order-of-magnitude higher accuracy is required is discussed in more details by Castro et al. (2017). Ideally, it should be possible to improve the measurements and the integral calculations by refining the positioning system (e.g., by employing an ultrasonic or laser detector and a camera to measure the local distances with respect to the ground and the buildings), even though this 
will affect the duration of the experiments. In the present study, though, the maximum horizontal distance from the nominal origin of the coordinate system is rather limited (the longest distance is $460 \mathrm{~mm}$ for Yst SS and Yst NN) and this factor is unlikely to have contributed much to the overall errors.

Whether a much higher resolution could improve the current results is debatable. The measurement grid shown in figure 1 is arguably very fine, with ten measurement points between buildings. This resolution should be enough to capture even the smaller significant scales (see also section 3). On the other hand, there is a physical limit on how close to the floor we can measure. This physical limit is dictated by laser reflection on the floor surface that, even though it can be reduced by painting the surface black, it could never be completely removed. As a matter of fact, laser light reflected by solid surfaces degrades the quality of LDA measurements and this is the reason why the lowest measurement point is at $z=20 \mathrm{~mm}$. The FFID does not suffer from this issue and so it was possible to measure at lower heights, down to $7 \mathrm{~mm}$ from the wind tunnel floor. Concentration maps for the $90^{\circ}$ cases are reported in figure 9 .

While the figures show a rather uniform concentration for the SimpleV case (upwind section Yst SS, the closest to the emission source), for the SimpleC case a more concentrated plume in the bottom part of the street canyon can be observed. In particular, the concentration peak appear to be located below the $20 \mathrm{~mm}$ height. This part of the plume is then undetected by the flux measurements and this is likely to be a major source of error for the integral flux calculated in the SimpleC $90^{\circ}$ case. It must be said, though, that the error in the Yst S street volume for this case is one of the lowest, with only a $2.40 \%$ imbalance between incoming and outgoing flux. This imbalance is also negative, meaning the calculated exiting flux is slightly larger than the entering flux. The underestimation of the incoming flux due to the error above is likely to be accountable for this imbalance.

As mentioned in section 1 the SimpleV case is completely different. The Yst S street volume for this case is the one with by far the largest calculated imbalance. As the concentration appears to be rather uniform, the interpolation algorithm is likely to approximate the actual flux pattern quite well in the bottom part of the section, where there are no velocity measurements available. Thus, this source of error does not appear to be very significant in this case.

\subsection{Blockage effect}

The large cross section of the EnFlo wind tunnel and a proper high-quality experimental methodology imply that blockage effects due to the instrumented traverse are kept to a minimum. On the other hand, the heavily instrumented traverse and the particular geometry (especially in the SimpleV case) of this study could have contributed to the errors in the calculations. For this reason a detailed study on the effect of traverse blockage on flow and concentration in the area of interest was undertaken. In particular the $90^{\circ}$ case was used.

A fixed velocity sensor not mounted on the moving traverse was placed within the Yst SS section while the fully instrumented traverse was moved around the measurement area. While the difference in the measured velocities 


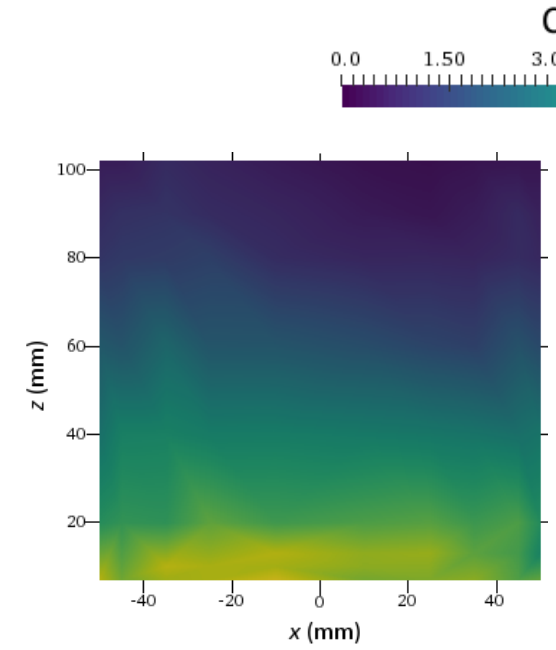

(a) SimpleC, Yst SS

$C^{*}$

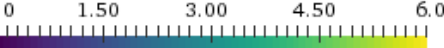

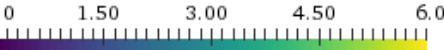

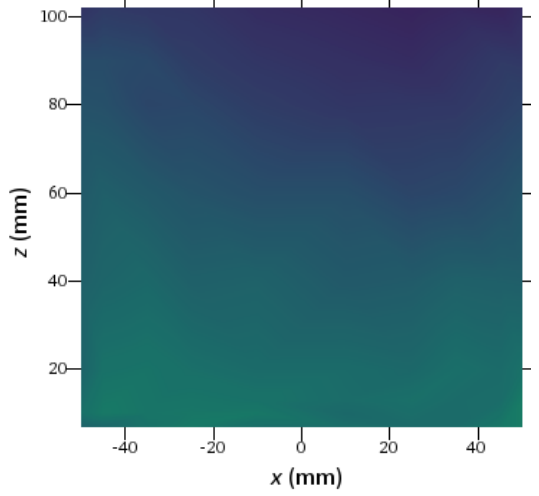

(b) SimpleC, Yst S

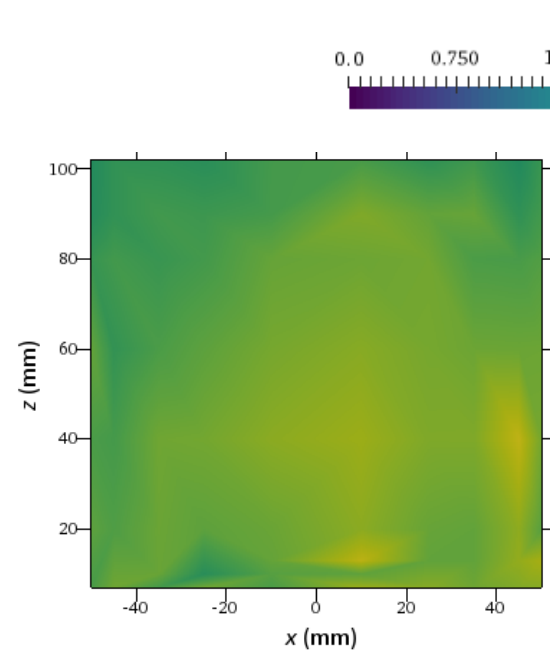

(c) SimpleV, Yst SS
$\mathrm{C}^{*}$

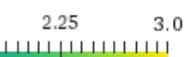

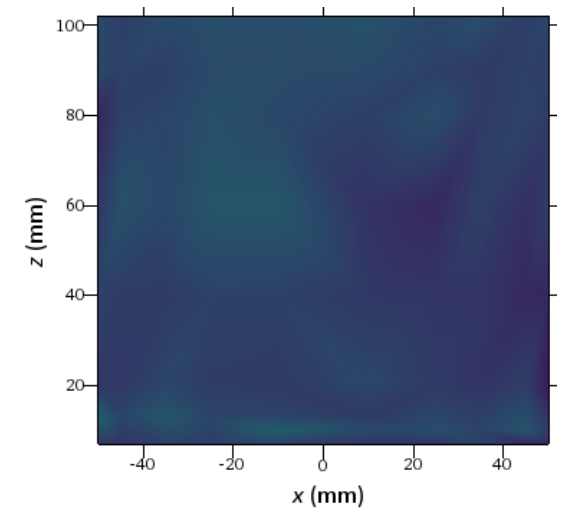

(d) SimpleV, Yst S

Figure 9: Non-dimensional mean concentrations for the $90^{\circ}$ cases 
at the fixed point in the SimpleC case were all below 5\%, a velocity increase of almost $16 \%$ was observed in the SimpleV case. The reason behind this different behavious can only be the different geometry between the two models, and in particular the presence of the two taller buildings close to where the traverse and the instrumentation are measuring. The same experiment was performed by measuring concentration at fixed points while moving the traverse around. In that case no significant differences were observed between the SimpleC and SimpleV models.

In summary, the large error in the flux balance for the SimpleV $90^{\circ}$ case was probably caused by an interaction between the traverse arm and the equipment it was carrying and the two taller buildings just upwind of the area of interest: they are closer to the instrumentation arm and the instrumentation itself, so that they cause some degree of a blockage effect (e.g. local flow acceleration) in the vicinity of the measurement gear. The blockage effect must have increased the measured velocity at some locations, leading to an overestimation of the total incoming flux. This acceleration issue does not affect the comparative study undertaken in the previous section, but it does impact on the accuracy of the flux balance for the SimpleV $90^{\circ}$ case. In all other cases, where the taller buildings are either not present or out of the flow path, the blockage effect is negligible.

\section{Conclusions}

This study presents wind tunnel measurements of mean and turbulent fluxes in an idealised urban area. As noted in the literature review, previous experimental studies of their properties in complex urban-like geometries are almost non-existent, while many recent mathematical models (in particular those adopting the street network approach) rely on some sort of flux parametrisations.

The methodology was initially tested on a model of a real urban area (Carpentieri et al., 2012). The present study attempted to further validate this methodology, while at the same time gaining more insight on the fundamental processes governing advective and turbulent mass exchanges within street canyons and between the urban canopy and the flow above. For this purpose a simpler geometry, yet one more realistic than regular arrays of cubes, was used.

As expected, horizontal advective fluxes were found to be dominant compared with the turbulent components. This is true for wind directions at an angle with respect to the street network (this study dealt in particular with the $45^{\circ}$ case) but also when the flow is parallel to the streets. This is an important result because it reduces the complexity in developing parametrisations for street network models, where horizontal exchanges between boxes (i.e. the street canyons and intersections) form a fundamental part of the modelling approach. This does not necessarily mean that this parametrisation will be simple. As matter of fact. previous studies (Carpentieri et al., 2009; Carpentieri \& Robins, 2010; Carpentieri et al., 2012) have shown how complex the mean mass flux can be in real urban areas, with large and counter-intuitive three-dimensional variations. The measurements presented here make an even stronger point due the fact 
that the studied geometry was much simpler, especially in the SimpleC case, but the results showed nevertheless large variations through the measurement sections. This variation is, of course, enhanced in the SimpleV case by different building heights upwind of the studied intersection (despite the fact that the intersection itself was the same in the two cases, to exclude very local effects).

On the other hand, vertical mean and turbulent fluxes through the top of the street canopy appear to be approximately of the same order of magnitude. Building height variability does not seem to affect the exchange process, while the presence of taller buildings upwind of the area of interest certainly does. In this case strong advective fluxes created by the wakes of the tall buildings overcome the weak turbulent fluxes. One of the most interesting results, again, is the fact that even very simple and regular geometries lead to complex advective patterns at intersections. This is true in the $45^{\circ}$ case but also and less expected in the $90^{\circ}$ case, where a narrow street crosses a much wider perpendicular street. Thus, parametrisations derived from measurements in simpler, regular geometries (even simpler than the ones studied here) are unlikely to capture the full complexity of a real urban area.

The above considerations must be taken into account when using single point measurements or single vertical profiles to validate mathematical parametrisations. Calculating the integral properties across the interface sections (street-to-street or street-to-above) is a non-trivial exercise and even mildly complex geometries may produce very complex spatial distribution of mean and turbulent fluxes. Current parameterisations were developed by applying many simplifications (see, e.g., Soulhac et al., 2011; Belcher et al., 2015) and are not able to capture the full complexity highlighted here.

Given the high resolution of the measurement grid, an attempt at integration was made to calculate a mass balance of the intersection, as in Carpentieri \& Robins (2010) and Carpentieri et al. (2012). However, despite the improved methodology and experimental strategy, an evaluation completely free of uncertainties was not possible. As discussed in section 5, an attempt at identifying the sources of these uncertainties was made. While the flux contributions from regions where measurements were impossible surely have an effect on the balance calculation (especially the area below $z=20 \mathrm{~mm}$ ), their effect was found to be not very large. The largest source of error seems to be the blockage effect arising from the combination of a heavily instrumented traverse gear with large obstacles such as the two taller buildings upwind of the central intersection in the SimpleV $90^{\circ}$ case. Such flows (i.e. perpendicular to the obstacles) are thought to be very sensitive to small perturbations and symmetry is most unlikely.

The above issue is hardly avoidable with the current methodology, and a case-by-case assessment needs to be implemented when designing experimental campaigns. Alternative experimental techniques could be used, for example by combining PIV and laser induced fluorescence (LIF, see for example Vanderwel \& Tavoularis, 2014: Vincont et al., 2000) in a water flume or Mie scattering diffusion (MSD, Vinçont et al., 2000). Of course, a totally different approach could be used: wind tunnel measurements could be integrated with high-resolution numerical models (e.g., large eddy simulations, LES, or direct numerical si- 
mulations, DNS). These computations do not have the constraints faced by laboratory experiments and, if properly validated, can accurately predict mean and turbulent mass transfer in the regions of interest. This is the approach attempted in the DIPLOS project (http://www.diplos.org), for example, with encouraging preliminary results (Castro et al., 2017).

\section{Acknowledgements}

This research has been funded by the European Commission under the FP7 People Programme, Marie-Curie Fellowship (PIEF-GA-2008-219318). ES work at the EnFlo laboratory has been partially funded by the European Commission under the Erasmus+ Programme.

\section{References}

Barlow, J. F., \& Belcher, S. E. (2002). A wind tunnel model for quantifying fluxes in the urban boundary layer. Boundary-Layer Meteorology, 104, 131150.

Barlow, J. F., Harman, I. N., \& Belcher, S. E. (2004). Scalar fluxes from urban street canyons. Part I: Laboratory simulation. Boundary-Layer Meteorology, 113, 369-385.

Belcher, S. E., Coceal, O., Goulart, E. V., Rudd, A. C., \& Robins, A. G. (2015). Processes controlling atmospheric dispersion through city centres. Journal of Fluid Mechanics, 763, 51-81.

Ben Salem, N., Garbero, V., Salizzoni, P., Lamaison, G., \& Soulhac, L. (2015). Modelling pollutant dispersion in a street network. Boundary-Layer Meteorology, 155, 157-187.

Carpentieri, M. (2013). Pollutant dispersion in the urban environment. Reviews in Environmental Science and Bio/Technology, 12, 5-8.

Carpentieri, M., Hayden, P., \& Robins, A. G. (2012). Wind tunnel measurements of pollutant turbulent fluxes in urban intersections. Atmospheric Environment, 46, 669-674.

Carpentieri, M., \& Robins, A. G. (2010). Tracer flux balance at an urban canyon intersection. Boundary-Layer Meteorology, 135, 229-242.

Carpentieri, M., \& Robins, A. G. (2015). Influence of urban morphology on air flow over building arrays. Journal of Wind Engineering and Industrial Aerodynamics, 145, 61-74.

Carpentieri, M., Robins, A. G., \& Baldi, S. (2009). Three-dimensional mapping of air flow at an urban canyon intersection. Boundary-Layer Meteorology, 133, 277-296. 
Castro, I. P., Xie, Z.-T., Fuka, V., Robins, A. G., Carpentieri, M., Hayden, P., Hertwig, D., \& Coceal, O. (2017). Measurements and computations of flow in an urban street system. Boundary-Layer Meteorology, 162, 207-230.

Caton, F., Britter, R. E., \& Dalziel, S. (2003). Dispersion mechanisms in a street canyon. Atmospheric Environment, 37, 693-702.

Dezső-Weidinger, G., Stitou, A., van Beeck, J., \& Riethmuller, M. L. (2003). Measurement of the turbulent mass flux with PTV in a street canyon. Journal of Wind Engineering and Industrial Aerodynamics, 91, 1117-1131.

Fackrell, J. E., \& Robins, A. G. (1982). Concentration fluctuations and fluxes in plumes from point sources in a turbulent boundary layer. Journal of Fluid Mechanics, 117, 1-26.

Gamel, H. (2015). Caractérisation expérimentale de l'écoulement et de la dispersion autour d'un obstacle bidimensionnel. PhD thesis École Centrale de Lyon.

Gamel, H., Salizzoni, P., Marro, M., Mejean, P., Carissimo, B., \& Soulhac, L. (2015). Dispersion of a passive scalar in the wake of a two-dimensional obstacle: velocity and concentration measurements. In PHYSMOD 2015, International workshop on physical modelling of flow and dispersion phenomena, Zurich, Switzerland, 7-9/9/2015.

Hoydysh, W. G., \& Dabberdt, W. F. (1994). Concentration fields at urban intersections: fluid modeling studies. Atmospheric Environment, 28, 18491860.

Lemoine, F., Wolff, M., \& Lebouché, M. (1997). Experimental investigation of mass transfer in a grid-generated turbulent flow using combined optical methods. International Journal of Heat and Mass Transfer, 40, 3255-3266.

McHugh, C., Carruthers, D., \& Edmunds, H. (1997). ADMS-Urban: an air quality management system for traffic, domestic and industrial pollution. International Journal of Environment and Pollution, 8, 666-674.

Narita, K.-i. (2007). Experimental study of the transfer velocity for urban surfaces with a water evaporation method. Boundary-Layer Meteorology, 122, 293-320.

Nosek, Š., Kukačka, L., Jurčáková, K., Kellnerová, R., \& Jaňour, Z. (2017). Impact of roof height non-uniformity on pollutant transport between a street canyon and intersections. Environmental Pollution, 227, 125-138.

Nosek, Š., Kukačka, L., Kellnerová, R., Jurčáková, K., \& Jaňour, Z. (2016). Ventilation processes in a three-dimensional street canyon. Boundary-Layer Meteorology, 159, 259-284. 
Pascheke, F., Barlow, J. F., \& Robins, A. (2008). Wind-tunnel modelling of dispersion from a scalar area source in urban-like roughness. Boundary-Layer Meteorology, 126, 103-124.

Salizzoni, P., Marro, M., Soulhac, L., Grosjean, N., \& Perkins, R. J. (2011). Turbulent transfer between street canyons and the overlying atmospheric boundary layer. Boundary-Layer Meteorology, 141, 393-414.

Scaperdas, A.-S. (2000). Modelling air flow and pollutant dispersion at urban canyon intersections. $\mathrm{PhD}$ thesis Imperial College London (University of London).

Soulhac, L., Salizzoni, P., Cierco, F. X., \& Perkins, R. (2011). The model SIRANE for atmospheric urban pollutant dispersion; part I, presentation of the model. Atmospheric Environment, 45, 7379-7395.

Tominaga, Y., \& Stathopoulos, T. (2013). CFD simulation of near-field pollutant dispersion in the urban environment: A review of current modeling techniques. Atmospheric Environment, 79, 716-730.

Vanderwel, C., \& Tavoularis, S. (2014). Measurements of turbulent diffusion in uniformly sheared flow. Journal of Fluid Mechanics, 754, 488-514.

Vinçont, J.-Y., Simoëns, S., Ayrault, M., \& Wallace, J. M. (2000). Passive scalar dispersion in a turbulent boundary layer from a line source at the wall and downstream of an obstacle. Journal of Fluid Mechanics, 424, 127-167.

Zhu, J. Y., So, R. M. C., \& Otugen, M. V. (1988). Turbulent mass flux measurements using a laser/hot-wire technique. International Journal of Heat and Mass Transfer, 31, 819-829. 